\title{
RANDOM FRACTALS GENERATED BY A LOCAL GAUSSIAN PROCESS INDEXED BY A CLASS OF FUNCTIONS
}

\author{
Claire Coiffard ${ }^{1}$
}

\begin{abstract}
In this paper, we extend the results of Orey and Taylor [S. Orey and S.J. Taylor, How often on a Brownian path does the law of the iterated logarithm fail? Proc. London Math. Soc. 28 (1974) 174-192] relative to random fractals generated by oscillations of Wiener processes to a multivariate framework. We consider a setup where Gaussian processes are indexed by classes of functions.
\end{abstract}

Mathematics Subject Classification. 60J65, 28A80.

Received November 25, 2008. Revised July 24, 2009.

\section{INTRODUCTION}

Let $\{W(t), t \in[0,1]\}$ denote a Wiener process. Lévy [8] studied the modulus of continuity of $W$, and obtained the following limiting law. We have

$$
\lim _{h \rightarrow 0} \sup _{0 \leq t \leq 1-h} \frac{W(t+h)-W(t)}{\sqrt{2 h \log (1 / h)}}=1, \quad \text { p.s. }
$$

This result shows that some points of a Brownian path don't follow the usual law of iterated logarithm. According to this law, for each fixed $t_{0} \in[0,1]$,

$$
\limsup _{h \rightarrow 0} \frac{W\left(t_{0}+h\right)-W\left(t_{0}\right)}{\sqrt{2 h \log \log h}}=1, \quad \text { p.s. }
$$

Orey and Taylor [11] introduced the random sets defined, for $\Lambda \in[0,1]$, by

$$
E_{\Lambda}=\left\{t \in[0,1]: \limsup _{h \downarrow 0}(2 h \log (1 / h))^{-1 / 2}(W(t+h)-W(t)) \geq \Lambda\right\} .
$$

For each $\Lambda>0 E_{\Lambda}$ collects the exceptional points in $[0,1]$ where the law of the iterated logarithm (1.2) fails. Orey and Taylor [11] showed that, with probability $1, E_{\Lambda}$ is a random fractal with Hausdorff dimension, given by

$$
\operatorname{dim} E_{\Lambda}=1-\Lambda^{2} .
$$

Keywords and phrases. Random fractals, Hausdorff dimension, Wiener process.

${ }^{1}$ LSTA, Université Pierre et Marie Curie, 175 rue du Chevaleret, 75013 Paris, France; claire.coiffard@centrale-marseille.fr 
Recall (see, e.g., Falconer [7]) that the Hausdorff dimension of $E \subset[0,1]$ is defined by

$$
\operatorname{dim} E=\inf \left\{c>0: s^{c}-\operatorname{mes}(E)=0\right\}=\sup \left\{c>0: s^{c}-\operatorname{mes}(E)=\infty\right\},
$$

where $s^{c}-\operatorname{mes}(E)$ denotes the Hausdorff measure of $\mathrm{E}$, given by

$$
s^{c}-\operatorname{mes}(E)=\lim _{\delta \rightarrow 0} \inf \left\{\sum_{i \geq 1}\left|U_{i}\right|^{c}: E \subseteq \bigcup_{i \geq 1} U_{i},\left|U_{i}\right| \leq \delta\right\} .
$$

We denote by $\left|U_{i}\right|$ the diameter of $U_{i}$, namely, the supremum of the Euclidean distance between two elements of $U_{i}$. The infimum in (1.4) is taken over all collections $\left\{U_{i}: i \geq 1\right\}$ of subsets with diameter $\left|U_{i}\right|<\delta$ for all $i \geq 1$ and such that $E \subseteq \bigcup_{i>1} U_{i}$. The identity (1.3) was extended in various directions. In particular, Deheuvels and Mason [4] and Deheuvels and Lifshits [3] established functional versions of (1.3). Dindar [6] extended this result to Wiener processes on $\mathbb{R}^{2}$. Our work will rely in part on the approach of Mason [10] where processes are indexed by class of functions.

The aim of this paper is to provide a largely extended version of (1.1) and (1.3) in the framework of multiparameter Gaussian processes, indexed by classes of functions. We start by giving some notation which is needed for the statement of our results.

We consider a class $\mathcal{F}$ of bounded functions on $I^{d}=[0,1]^{d}$. We denote by $\|f\|$ the sup-norm of the function $f \in \mathcal{F}$. Let $|\cdot|_{2}$ denote the usual Euclidean norm on $\mathbb{R}^{d}$. Assume that the class $\mathcal{F}$ satisfies :

F.i. $\lim _{|\mathbf{w}|_{2} \rightarrow 0} \sup _{f \in \mathcal{F}} \int_{\mathbb{R}^{d}}(f(\mathbf{x})-f(\mathbf{x}+\mathbf{w}))^{2} \mathrm{~d} \mathbf{x}=0$,

F.ii. $\lim _{\lambda \rightarrow 1} \sup _{f \in \mathcal{F}} \int_{\mathbb{R}^{d}}(f(\mathbf{x})-f(\lambda \mathbf{x}))^{2} \mathrm{~d} \mathbf{x}=0$

F.iii. for each $\lambda \geq 1, \mathbf{z} \in \mathbb{R}^{d}$ and $f \in \mathcal{F}, f(\mathbf{z}-\lambda \cdot) \in \mathcal{F}$,

F.iv. $\mathcal{F}$ is a $V C$-subgraph class,

F.v. $\mathcal{F}$ is pointwise measurable class. That is, there exists a countable subclass $\mathcal{F}_{0}$ of $\mathcal{F}$ such that for each function $f \in \mathcal{F}$, there exists a sequence of functions $\left\{f_{m}\right\} \in \mathcal{F}_{0}$ such that $f_{m}(z) \rightarrow f(z), z \in \mathbb{R}^{d}$.

A collection of measurable functions $\mathcal{F}$ on a sample space $\mathcal{X}$ is called a $V C$-subgraph class if the collection of all subgraphs of the functions in $\mathcal{F}$ forms a $V C$-class of sets in $\mathcal{X} \times \mathbb{R}$. For a definition of a $V C$-class or Vapnik-Cernonenkis class, we refer to p. 141 in Van der Vaart and Wellner [14]. A VC-class satisfies an entropy condition, of the following type. For each $\varepsilon>0$, the covering number $N(\varepsilon, \mathcal{F},\|\cdot\|)$ is the minimal number of balls $\{g:\|g-f\|<\varepsilon\}$ of radius $\varepsilon$ needed to cover the set $\mathcal{F}$. For some $A>0$, the covering number $N(\varepsilon, \mathcal{F},\|\cdot\|)$ of a $\mathrm{VC}$-class $\mathcal{F}$ grows polynomially in $A / \varepsilon$ as $\varepsilon \downarrow 0$.

The multivariate local Gaussian process at $\mathbf{z} \in \mathbb{R}^{d}$, indexed by $f \in \mathcal{F}$, is defined by

$$
W(h, \mathbf{z} ; f)=\int_{\mathbb{R}^{d}} f\left(\frac{\mathbf{z}-\mathbf{u}}{h^{1 / d}}\right) \mathrm{d} W(\mathbf{u}),
$$

where $W(\mathbf{z}), \mathbf{z} \in \mathbb{R}^{d}$ denotes a standard Wiener process with $d$ parameters in $\mathbb{R}^{d}$. We set

$$
\Theta_{h, \mathbf{z}}(f)=\frac{W(h, \mathbf{z} ; f)}{\sqrt{2 h \log (1 / h)}}=\frac{\int_{\mathbb{R}^{d}} f\left(h^{-1 / d}(\mathbf{z}-\mathbf{u})\right) \mathrm{d} W(\mathbf{u})}{\sqrt{2 h \log (1 / h)}} .
$$

We define an inner product of $f_{1}, f_{2} \in \mathcal{F}$ by setting

$$
\left\langle f_{1}, f_{2}\right\rangle_{L_{2}}:=\int_{I^{d}} f_{1}(\mathbf{u}) f_{2}(\mathbf{u}) \mathrm{d} \mathbf{u} .
$$


Let $G_{2}(\mathcal{F})$ be the Hilbert subspace of $L_{2}(\mathcal{F})$ spanned by $\mathcal{F}$. For each $\varphi \in G_{2}(\mathcal{F})$, we denote by $\Theta_{\varphi}$ the functional $\Theta_{\varphi}(f)=\int_{I^{d}} f(\mathbf{u}) \varphi(\mathbf{u})$ du. For each $\Lambda \in[0,1]$, we set

$$
\mathcal{H}_{\Lambda}=\left\{\Theta_{\varphi}(f), f \in \mathcal{F}, \varphi \in G_{2}(\mathcal{F}) \text { with } \int_{I^{d}} \varphi^{2}(\mathbf{u}) \mathrm{d} \mathbf{u} \leq \Lambda^{2}\right\} .
$$

Let $\ell_{\infty}(\mathcal{F})$ denote the class of bounded functions on $\mathcal{F}$, endowed with the sup-norm $\|\cdot\|_{\mathcal{F}}$. For any $\vartheta \in \mathcal{H}_{1}$ and $\varepsilon>0$, set

$$
B_{\varepsilon}(\vartheta)=\left\{\psi \in \ell_{\infty}(\mathcal{F}):\|\psi-\vartheta\|_{\mathcal{F}}<\varepsilon\right\}
$$

Finally, for any $\varepsilon>0$, we set

$$
\mathcal{H}_{1}^{\varepsilon}=\left\{\psi \in \ell_{\infty}(\mathcal{F}): \inf _{\vartheta \in \mathcal{H}_{1}}\|\psi-\vartheta\|_{\mathcal{F}}<\varepsilon\right\}
$$

The following result gives a uniform functional law of the logarithm for local Gaussian processes indexed by a class of functions.

Theorem A. Let $J$ be a compact subset of $\mathbb{R}^{d}$ with nonempty interior. Suppose that (F.i-v) hold, and set for $\gamma>0$

$$
h_{k}^{-1}=\left\lfloor(1+\gamma)^{k}\right\rfloor
$$

Then, with probability 1 ,

(a) for each $\varepsilon>0$, there exists a $\delta$ such that, for all $0<h \leq \delta,\left\{\Theta_{h, \mathbf{z}}: \mathbf{z} \in J\right\} \subset \mathcal{H}_{1}^{\varepsilon}$,

(b) for each $\vartheta \in \mathcal{H}_{1}$ and $\varepsilon>0$, there exists a $k(\vartheta, \varepsilon)$ such that for all $k \geq k(\vartheta, \varepsilon)$, there exists a $\mathbf{z}_{k} \in J$ such that $\Theta_{h_{k}, \mathbf{z}_{k}} \in B_{\varepsilon}(\vartheta)$.

Proof. The proof of this theorem is given in Mason [9].

Now for each $\varphi \in G_{2}(\mathcal{F})$, following the lines of Orey and Taylor [11], we consider the set of points defined by

$$
L\left(\Theta_{\varphi}\right)=\left\{\mathbf{z} \in I^{d}: \liminf _{h \downarrow 0}\left\|\Theta_{h, \mathbf{z}}-\Theta_{\varphi}\right\|_{\mathcal{F}}=0\right\} .
$$

This set collects the points of $I^{d}$ where $\Theta_{h^{d}, \mathbf{z}}$ is infinitely often in a neighborhood of the function $\Theta_{\varphi}$. Set further, for $\Lambda \geq 0$

$$
L_{\Lambda}=\bigcup\left\{L\left(\Theta_{\varphi}\right), \Theta_{\varphi} \in \mathcal{H}_{1}, \varphi \in G_{2}(\mathcal{F}), \int_{I^{d}} \varphi^{2}(\mathbf{u}) \mathrm{d} \mathbf{u} \geq \Lambda^{2}\right\} .
$$

Our main result, stated in the following theorem, evaluates the Hausdorff dimensions of $L\left(\Theta_{\varphi}\right)$ and $L_{\Lambda}$.

Theorem 1.1. Assume that $\mathcal{F}$ fulfills $F . i-v$, and let $\varphi \in G_{2}(\mathcal{F})$ be such that

$$
\int_{I^{d}} \varphi^{2}(\boldsymbol{u}) \mathrm{d} \boldsymbol{u} \leq 1
$$

Then for each $\Lambda \in[0,1]$, the sets $L\left(\Theta_{\varphi}\right)$ and $L_{\Lambda}$ are almost surely everywhere dense in $I^{d}$ and satisfy

$$
\operatorname{dim} L\left(\Theta_{\varphi}\right)=d\left(1-\int_{I^{d}} \varphi^{2}(\boldsymbol{u}) \mathrm{d} \boldsymbol{u}\right) \text { and } \operatorname{dim} L_{\Lambda}=d\left(1-\Lambda^{2}\right) .
$$

The proof of Theorem 1.1 is based on an adaptation of the arguments of Deheuvels and Mason [5] and Deheuvels and Lifshits [3] and is given in Section 2. 


\section{Proof of Theorem 1.1}

Let the assumptions of Theorem 1.1 be in force. The proof of (1.11) reduces to show that with probability 1 , for all $0 \leq \Lambda \leq 1$,

and, for all $\Theta_{\varphi} \in \mathcal{H}_{1}$ and $\varphi \in G_{2}(\mathcal{F})$,

$$
\operatorname{dim} L_{\Lambda} \leq d\left(1-\Lambda^{2}\right)
$$

$$
\operatorname{dim} L\left(\Theta_{\varphi}\right) \geq d\left(1-\int_{I^{d}} \varphi^{2}(\mathbf{u}) \mathrm{d} \mathbf{u}\right)
$$

To establish (2.1) and (2.2) we will need the preliminary facts given in the next section. In Section 2.2 we will establish (2.1) and in Section 2.3 we will provide a proof for (2.2).

\subsection{Preliminary facts}

Fact 2.1 below is a generalization of the well-known result of Schilder [12] relative to large deviations. For any $\psi \in \ell_{\infty}(\mathcal{F})$, we set

$$
I(\psi)= \begin{cases}\frac{1}{2} \int_{I^{d}} \xi^{2}(\mathbf{u}) \mathrm{d} \mathbf{u} & \text { when } \psi(f)=\langle f, \xi\rangle_{L_{2}} \text { for some } \xi \in G_{2}(\mathcal{F}) \\ \infty & \text { otherwise. }\end{cases}
$$

Also, for any subset $B \subset \ell_{\infty}(\mathcal{F})$, we set

$$
I(B)=\inf \{I(\psi): \psi \in B\}
$$

Fact 2.1. Let $\mathcal{F}$ be a class of functions fulfilling Fi-Fii, and let $\left\{\varpi_{k}: k \geq 1\right\}$ be a sequence of constants such that $\varpi_{k} \rightarrow 0$ when $k \rightarrow \infty$. Set $\varepsilon_{k}=\left(2 \log \left(1 / \varpi_{k}\right)\right)^{-1}$, for $k=0,1, \ldots$ Then,

(i) for each closed subset $F$ of $\ell_{\infty}(\mathcal{F})$

$$
\limsup _{k \rightarrow \infty} \varepsilon_{k} \log P\left\{\Theta_{\varpi_{k}, z} \in F\right\} \leq-I(F),
$$

(ii) for each open subset $G$ of $\ell_{\infty}(\mathcal{F})$

$$
\liminf _{k \rightarrow \infty} \varepsilon_{k} \log P\left\{\Theta_{\varpi_{k}, z} \in G\right\} \geq-I(G) .
$$

Proof. It follows readily from Theorem 5.2 in Arcones [1]. (This same method is used for the proof of Prop. 1 in Mason [10].)

The next fact will be instrumental in the proof of $(2.2)$.

Fact 2.2. Let $A \subseteq I^{d}$ be such that $A=\bigcap_{m=1}^{\infty} E_{m}$, where $E_{1} \supseteq E_{2} \supseteq \ldots$ and $E_{m}=\bigcup_{k=1}^{M_{m}} J_{m, k}$, with $\left\{J_{m, k}\right.$ : $\left.1 \leq k \leq M_{m}\right\}$ being for each $m \geq 1$, a collection of disjoint hypercubes of $\mathbb{R}^{d}$, such that $\max _{1 \leq k \leq M_{m}}\left|J_{m, k}\right| \rightarrow 0$ and $M_{m} \rightarrow \infty$ as $m \rightarrow \infty$. Then, whenever there exist two constants $\Delta>0$ and $\delta>0$ such that, for every hypercube $J \subseteq I^{d}$ with $|J| \leq \Delta$, there is a constant $m(J)$ such that for all $m \geq m(J)$

$$
M_{m}(J):=\#\left\{J_{m, k} \subseteq J: 1 \leq k \leq M_{m}\right\} \leq \delta|J|^{c} M_{m}
$$

we have $s^{c}-\operatorname{mes}(A)>0$.

Proof. See, e.g., Lemma 2.2 of Orey and Taylor [11].

The next fact gives a useful property of the binomial distribution. 
Fact 2.3. Let $S_{N}$ follow a binomial distribution with parameters $N$ and $p$. Then, for all $r \in[1, \infty]$,

$$
\mathbb{P}\left(S_{N} \geq N r p\right) \leq \exp (-N p h(r))
$$

and for all $r \in[0,1]$,

$$
\mathbb{P}\left(S_{N} \leq N r p\right) \leq \exp (-N p h(r))
$$

where $h$ is the Chernoff function associated with the standard Poisson process and defined by

$$
h(r)= \begin{cases}r \log r-r+1 & \text { for } r>0 \\ 1 & \text { for } r=0 \\ \infty & \text { otherwise }\end{cases}
$$

Proof. The proof of (2.6) and (2.7) is based on Markov inequalities (See, e.g., Chernoff [2]). This result is in Lemma 3.8 of Deheuvels and Mason [5]).

\subsection{Upper bounds}

In this part, we establish (2.1). Actually, it is sufficient to show the result for $0<\Lambda<1$. Indeed, if $\Lambda_{1} \leq \Lambda_{2}$, $L_{\Lambda_{2}} \subseteq L_{\Lambda_{1}}$ and by the properties of the Hausdorff dimension, $\operatorname{dim} L_{\Lambda_{2}} \leq \operatorname{dim} L_{\Lambda_{1}}$. So, for all $0<\Lambda<1$, $\operatorname{dim} L_{1} \leq d\left(1-\Lambda^{2}\right)$. Since $\Lambda \in(0,1)$ is arbitrary, $\operatorname{dim} L_{1}=0$. The inequality $\operatorname{dim} L_{0} \leq d$ is trivial, and so, it is enough to prove $(2.1)$ for $0<\Lambda<1$.

In order to establish (2.1), we must first fix some notation. Set

$$
L_{\Lambda^{+}}=\bigcup\left\{L\left(\Theta_{\varphi}\right), \Theta_{\varphi} \in \mathcal{H}_{1}, \varphi \in G_{2}(\mathcal{F}), \int_{I^{d}} \varphi^{2}(\mathbf{u}) \mathrm{d} \mathbf{u}>\Lambda^{2}\right\}
$$

Remember that for every set $\mathcal{G}$, the neighborhood of $\mathcal{G}$ in $\ell_{\infty}(\mathcal{F})$ is defined by

$$
\mathcal{G}^{\varepsilon}=\left\{\phi \in \ell_{\infty}(\mathcal{F}): \inf _{\vartheta \in \mathcal{G}}\|\phi-\vartheta\|_{\mathcal{F}}<\varepsilon\right\}
$$

We set

$$
L(h, \varepsilon, \Lambda)=\left\{\mathbf{z} \in I^{d}: \Theta_{h, \mathbf{z}} \notin \mathcal{H}_{\Lambda}^{\varepsilon}\right\}
$$

and

$$
L(\varepsilon, \Lambda)=\left\{\mathbf{z} \in I^{d}: \Theta_{h, \mathbf{z}} \notin \mathcal{H}_{\Lambda}^{\varepsilon} \text { i.o }\right\} .
$$

We can see that for all $\Lambda>0$ and for all integer $m_{0} \geq 1$, we have

$$
L_{\Lambda^{+}} \subseteq \bigcup_{m=m_{0}}^{\infty} L(1 / m, \Lambda)
$$

Therefore, in order to establish (2.1), it is enough to show that for all $0<\Lambda<1$ and $\varepsilon>0$,

$$
s^{d\left(1-\Lambda^{2}\right)}-\operatorname{mes}(L(\varepsilon, \Lambda))<\infty .
$$

The following lemma will be crucial to control the oscillations between two points. For $f \in \mathcal{F}$, consider the process

$$
Y(f)=\int_{\mathbb{R}^{d}} f(\mathbf{u}) \mathrm{d} W(\mathbf{u}) .
$$


Notice that for $f_{1}, f_{2} \in \mathcal{F}$, the usual pseudo metric between $f_{1}$ and $f_{2}$ is defined by

$$
\rho\left(f_{1}, f_{2}\right)=\sqrt{E\left(Y\left(f_{1}\right)-Y\left(f_{2}\right)\right)^{2}}=\sqrt{\int_{\mathbb{R}^{d}}\left(f_{1}-f_{2}\right)^{2}(\mathbf{u}) \mathrm{d} \mathbf{u} .}
$$

Lemma 2.4. There exists a function $\psi(\delta)$ of $\delta>0$, fulfilling $\psi(\delta) \rightarrow 0$ as $\delta \downarrow 0$, and such that

$$
\limsup _{h \downarrow 0} \sup _{\rho\left(f_{1}, f_{2}\right) \leq \sqrt{\delta h}} \frac{\left|Y\left(f_{1}\right)-Y\left(f_{2}\right)\right|}{\sqrt{2 h \log (1 / h)}}=\psi(\delta) \quad \text { a.s. }
$$

Proof. See, e.g., Mason [9].

Remember that $|\cdot|_{2}$ stands for the Euclidean norm. By F.i-ii, there exists a function $A(\delta), \delta>0$, such that

$$
\begin{gathered}
\lim _{\delta \rightarrow 0} A(\delta)=0, \\
\sup _{f \in \mathcal{F}} \sup _{|\mathbf{w}|_{2} \leq \delta} \int_{\mathbb{R}^{d}}(f(\mathbf{x})-f(\mathbf{x}+\mathbf{w}))^{2} \mathrm{~d} \mathbf{x} \leq A(\delta) . \\
\sup _{f \in \mathcal{F}} \sup _{1-\delta \leq \lambda \leq 1+\delta} \int_{\mathbb{R}^{d}}(f(\mathbf{x})-f(\lambda \mathbf{x}))^{2} \mathrm{~d} \mathbf{x} \leq A(\delta) .
\end{gathered}
$$

The following lemma will be needed to apply Lemma 2.4 in our proofs.

Lemma 2.5. For all $0<\delta<1$, and $\mathbf{t}, \mathbf{t}^{\prime} \in I^{d}$ and $h, h^{\prime}>0$,

$$
\sup _{f \in \mathcal{F}} \sup _{\left|\mathbf{t}-\mathbf{t}^{\prime}\right|_{2} \leq \delta h^{1 / d}} E\left(W(h, \mathbf{t} ; f)-W\left(h, \mathbf{t}^{\prime} ; f\right)\right)^{2} \leq A(\delta) h .
$$

and

$$
\sup _{f \in \mathcal{F}} \sup _{(1-\delta)^{d} \leq h / h^{\prime} \leq(1+\delta)^{d}} E\left(W(h, \mathbf{t} ; f)-W\left(h^{\prime}, \mathbf{t} ; f\right)\right)^{2} \leq A(\delta) h .
$$

Proof. We start with (2.15). Observe that

$$
\begin{aligned}
E\left(W(h, \mathbf{t} ; f)-W\left(h, \mathbf{t}^{\prime} ; f\right)\right)^{2} & =E\left(\int_{\mathbb{R}^{d}} f\left(\frac{\mathbf{t}-\mathbf{u}}{h^{1 / d}}\right)-f\left(\frac{\mathbf{t}^{\prime}-\mathbf{u}}{h^{1 / d}}\right) \mathrm{d} W(\mathbf{u})\right)^{2} \\
& =\int_{\mathbb{R}^{d}}\left[f\left(\frac{\mathbf{t}-\mathbf{u}}{h^{1 / d}}\right)-f\left(\frac{\mathbf{t}^{\prime}-\mathbf{u}}{h^{1 / d}}\right)\right]^{2} \mathrm{~d} \mathbf{u} \\
& =h \int_{\mathbb{R}^{d}}\left[f(\mathbf{x})-f\left(\mathbf{x}+\frac{\mathbf{t}^{\prime}-\mathbf{t}}{h^{1 / d}}\right)\right]^{2} \mathrm{~d} \mathbf{x} .
\end{aligned}
$$

Since $\left|h^{-1 / d}\left(\mathbf{t}^{\prime}-\mathbf{t}\right)\right|_{2} \leq \delta$, we infer (2.15) from (2.13). The proof of (2.16) is very similar, since

$$
\begin{aligned}
E\left(W(h, \mathbf{t} ; f)-W\left(h^{\prime}, \mathbf{t} ; f\right)\right)^{2} & =\int_{\mathbb{R}^{d}}\left[f\left(\frac{\mathbf{t}-\mathbf{u}}{h^{1 / d}}\right)-f\left(\frac{\mathbf{t}-\mathbf{u}}{h^{\prime / d}}\right)\right]^{2} \mathrm{~d} \mathbf{u} \\
& =h \int_{\mathbb{R}^{d}}\left(f(\mathbf{x})-f\left(\left(h / h^{\prime}\right)^{1 / d} \mathbf{x}\right)^{2}\right) \mathrm{d} \mathbf{x} .
\end{aligned}
$$

Given this relation, we infer (2.16) from (2.14) in order to obtain (2.16). 
Denote by $\lfloor x\rfloor \leq x<\lfloor x\rfloor+1$ the integer part of $x$. For $\gamma>0$, set

$$
\nu_{k}=\left\lfloor(1+\gamma)^{k / d}\right\rfloor, k=1,2, \ldots
$$

and for some integer $K \geq 1$ and for every $k \geq 1$, set

$$
\tau_{k}\left(i_{r}, K\right)=\frac{i_{r}}{K \nu_{k}} \text { for } 0 \leq i_{r} \leq K \nu_{k} \text { and } 1 \leq r \leq d
$$

In the multivariate framework, we use the notation $\mathbf{i}=\left(i_{1}, \ldots, i_{d}\right)$ and we set

$$
\tau_{k}(\mathbf{i}, K)=\left(\tau_{k}\left(i_{1}, K\right), \ldots, \tau_{k}\left(i_{d}, K\right)\right) .
$$

Finally, we also set

$$
\begin{aligned}
\mathbb{1}_{i_{1}, \ldots, i_{d}, k}(\varepsilon, \Lambda) & =\mathbb{1}\left\{\Theta_{1 / \nu_{k}^{d}, \tau_{k}(\mathbf{i}, K)} \notin \mathcal{H}_{\Lambda}^{\varepsilon / 2}\right\}, \\
I_{i_{1}, \ldots, i_{d}, k}(\varepsilon) & = \begin{cases}\prod_{r=1}^{d}\left[\frac{\tau_{k}\left(i_{r}-1, K\right)}{\sqrt{d}}, \frac{\tau_{k}\left(i_{r}+1, K\right)}{\sqrt{d}}\left[\begin{array}{l}
\text { if } \mathbb{1}_{i_{1}, \ldots, i_{d}, k}(\varepsilon, \Lambda)=1, \\
\varnothing
\end{array}\right.\right. & \text { otherwise. }\end{cases}
\end{aligned}
$$

Lemma 2.6. For all $K \geq 1$ large enough, there exists almost surely an $N<\infty$ such that for all $k \geq N$,

$$
V_{k}(\varepsilon, \Lambda):=\bigcup_{1 / \nu_{k}^{d} \leq h<1 / \nu_{k-1}^{d}} L(h, \varepsilon, \Lambda) \subseteq \bigcup_{0 \leq i_{1} \leq K \nu_{k}} \ldots \bigcup_{0 \leq i_{d} \leq K \nu_{k}} I_{i_{1}, \ldots, i_{d}, k}(\varepsilon),
$$

Proof. Let $\mathbf{t} \in \bigcup_{1 / \nu_{k}^{d} \leq h<1 / \nu_{k-1}^{d}} L(h, \varepsilon, \Lambda)$. Then, $\Theta_{h, \mathbf{t}} \notin \mathcal{H}_{\Lambda}^{\varepsilon}$ for some $h \in\left[1 / \nu_{k}^{d}, 1 / \nu_{k-1}^{d}[\right.$, which means that for any $\Theta_{\varphi} \in \mathcal{H}_{\Lambda}$,

$$
\left\|\Theta_{h, \mathbf{t}}-\Theta_{\varphi}\right\|_{\mathcal{F}}>\varepsilon \text { for some } h \in\left[1 / \nu_{k}^{d}, 1 / \nu_{k-1}^{d}[\text {. }\right.
$$

We choose $\mathbf{i}=\left(i_{1}, \ldots, i_{d}\right)$ such that $\mathbf{t} \in \prod_{r=1}^{d}\left[\frac{\tau_{k}\left(i_{r}-1, K\right)}{\sqrt{d}}, \frac{\tau_{k}\left(i_{r}+1, K\right)}{\sqrt{d}}[\right.$. Therefore, by triangular inequality,

$$
\left\|\Theta_{1 / \nu_{k}^{d}, \tau_{k}(\mathbf{i}, K)}-\Theta_{\varphi}\right\|_{\mathcal{F}} \geq\left\|\Theta_{h, \mathbf{t}}-\Theta_{\varphi}\right\|_{\mathcal{F}}-\left\|\Theta_{h, \mathbf{t}}-\Theta_{h, \tau_{k}(\mathbf{i}, K)}\right\|_{\mathcal{F}}-\left\|\Theta_{h, \tau_{k}(i, K)}-\Theta_{1 / \nu_{k}^{d}, \tau_{k}(i, K)}\right\|_{\mathcal{F}}
$$

We first bound the term $\left\|\Theta_{h, \mathbf{t}}-\Theta_{h, \tau_{k}(\mathbf{i}, K)}\right\|_{\mathcal{F}}$. Recalling $(2.19)$, we see that

$$
\left|\mathbf{t}-\tau_{k}(\mathbf{i}, K)\right|_{2} \leq \frac{2}{K \nu_{k}}
$$

By applying Lemma 2.5 to $\delta=2 / K$, we get

$$
\sup _{f \in \mathcal{F}} E\left(W(h, \mathbf{t} ; f)-W\left(h, \tau_{k}(\mathbf{i}, K) ; f\right)\right)^{2} \leq A\left(\frac{2}{K}\right) 1 / \nu_{k}^{d} .
$$

Notice that the function $b(h)=\sqrt{2 h \log (1 / h)}$ is increasing for $0<h<\mathrm{e}^{-1}$. We infer from Lemma 2.4 that for some $1 / \nu_{k}^{d} \leq h<1 / \nu_{k-1}^{d}$, there exists a function $\psi(\delta)$ of $\delta>0$, such that $\psi(\delta) \rightarrow 0$ as $\delta \downarrow 0$, and

$$
\begin{aligned}
\limsup _{k \rightarrow \infty} \sup _{f \in \mathcal{F}} \frac{\left|W(h, \mathbf{t} ; f)-W\left(h, \tau_{k}(\mathbf{i}, K) ; f\right)\right|}{\sqrt{2 h \log (1 / h)}} & =\limsup _{k \rightarrow \infty} \sup _{\rho\left(f_{1}, f_{2}\right) \leq \sqrt{A\left(\frac{2 d}{K}\right) \nu_{k}^{-d}}} \frac{\left|Y\left(f_{1}\right)-Y\left(f_{2}\right)\right|}{\sqrt{2 h \log (1 / h)}} \\
& \leq \limsup _{k \rightarrow \infty} \sup _{\rho\left(f_{1}, f_{2}\right) \leq \sqrt{A\left(\frac{2}{K}\right) \nu_{k}^{-d}}} \frac{\left|Y\left(f_{1}\right)-Y\left(f_{2}\right)\right|}{\sqrt{2 \nu_{k}^{-d} \log \left(\nu_{k}^{d}\right)}} \leq \psi\left(A\left(\frac{2}{K}\right)\right) .
\end{aligned}
$$


Then, for all $K \geq 1$ large enough, there exists almost surely an $N<\infty$ such that for all $k \geq N$,

$$
\left\|\Theta_{h, \mathbf{t}}-\Theta_{h, \tau_{k}(\mathbf{i}, K)}\right\|_{\mathcal{F}} \leq \varepsilon / 4 .
$$

We next provide an upper bound for $\left\|\Theta_{h, \tau_{k}(i, K)}-\Theta_{1 / \nu_{k}^{d}, \tau_{k}(i, K)}\right\|_{\mathcal{F}}$.

Recall that $1 / \nu_{k}^{d} \leq h \leq 1 / \nu_{k-1}^{d}$. Thus, using (2.16) in Lemmas 2.5, and 2.4, we find that for $K \geq 1$ large enough, there exists almost surely an $N<\infty$ such that for all $k \geq N$,

$$
\left\|\Theta_{h, \tau_{k}(i, K)}-\Theta_{1 / \nu_{k}^{d}, \tau_{k}(i, K)}\right\|_{\mathcal{F}} \leq \varepsilon / 4 \text {. }
$$

Next, we infer from (2.21), (2.22) and (2.23) that

$$
\left\|\Theta_{1 / \nu_{k}^{d}, \tau_{k}(\mathbf{i}, K)}-\Theta_{\varphi}\right\|_{\mathcal{F}} \geq \varepsilon / 2 .
$$

Finally, (2.18) and (2.24) jointly imply (2.20).

We now turn to the proof of $(2.1)$. For all $\Lambda \in(0,1)$ we have

$$
s^{d\left(1-\Lambda^{2}\right)}-\operatorname{mes}\left(V_{k}(\varepsilon, \Lambda)\right) \leq \sum_{i_{1}=0}^{K \nu_{k}} \ldots \sum_{i_{d}=0}^{K \nu_{k}}\left(\frac{2}{K \nu_{k}}\right)^{d\left(1-\Lambda^{2}\right)} \mathbb{1}_{i_{1}, \ldots, i_{d}, k}(\varepsilon, \Lambda),
$$

and so

$$
\begin{aligned}
s^{d\left(1-\Lambda^{2}\right)}-\operatorname{mes}\left(L_{\Lambda^{+}}\right) & \leq \sum_{k \geq 1}\left\{s^{d\left(1-\Lambda^{2}\right)}-\operatorname{mes}\left(V_{k}(\varepsilon, \Lambda)\right)\right\} \\
& \leq \sum_{k \geq 1} \sum_{i_{1}=0}^{K \nu_{k}} \ldots \sum_{i_{d}=0}^{K \nu_{k}}\left(\frac{2}{K \nu_{k}}\right)^{d\left(1-\Lambda^{2}\right)} \mathbb{1}_{i_{1}, \ldots, i_{d}, k}(\varepsilon, \Lambda) .
\end{aligned}
$$

We set $S_{k}=\sum_{i_{1}=0}^{K \nu_{k}} \ldots \sum_{i_{d}=0}^{K \nu_{k}} \mathbb{1}_{i_{1}, \ldots, i_{d}, k}(\varepsilon, \Lambda)$. To establish (2.1), it is enough to show that

$$
\sum_{k=1}^{\infty} \mathbb{E}\left\{\sum_{i_{1}=0}^{K \nu_{k}} \ldots \sum_{i_{d}=0}^{K \nu_{k}}\left(\frac{2}{K \nu_{k}}\right)^{d\left(1-\Lambda^{2}\right)} \mathbb{1}_{i_{1}, \ldots, i_{d}, k}(\varepsilon, \Lambda)\right\} \leq \sum_{k=1}^{\infty}\left(\frac{2}{K \nu_{k}}\right)^{d\left(1-\Lambda^{2}\right)} \mathbb{E} S_{k}<\infty .
$$

Observe that

$$
\begin{aligned}
\mathbb{E} S_{k} & =\sum_{i_{1}=0}^{K \nu_{k}} \ldots \sum_{i_{d}=0}^{K \nu_{k}} P\left(\Theta_{\left(1 / \nu_{k}\right)^{d}, \tau_{k}(\mathbf{i}, K)} \notin \mathcal{H}_{\Lambda}^{\varepsilon / 2}\right) \\
& =\left(K \nu_{k}+1\right)^{d} P\left(\Theta_{\left(1 / \nu_{k}\right)^{d}, \mathbf{0}} \notin \mathcal{H}_{\Lambda}^{\varepsilon / 2}\right),
\end{aligned}
$$

with $\mathbf{0}=(0, \ldots, 0)$. We now use Fact 2.1, with $\varpi_{k}=\left(1 / \nu_{k}\right)^{d}$ and $F=\ell_{\infty}(\mathcal{F})-\mathcal{H}_{\Lambda}^{\varepsilon / 2}$. Obviously, for $\rho>0$, $2 I(F) \geq \Lambda^{2}+\rho$. We therefore obtain

$$
\begin{aligned}
\mathbb{E} S_{k} & \leq\left(K \nu_{k}+1\right)^{d} \exp \left\{-2 d \log \nu_{k}\left(\Lambda^{2}+\rho\right) / 2\right\} \\
& \leq\left(K \nu_{k}+1\right)^{d} \nu_{k}^{-d\left(\Lambda^{2}+\rho\right)} .
\end{aligned}
$$

Thus,

$$
\left(\frac{2}{K \nu_{k}}\right)^{d\left(1-\Lambda^{2}\right)} \mathbb{E} S_{k}=(1+o(1)) \nu_{k}^{-d \rho}=(1+o(1))(1+\gamma)^{-k \rho} .
$$

We have shown that (2.25), and hence (2.1), holds. 


\subsection{Lower bounds}

In this section, we will establish that for every $\Theta_{\varphi} \in \mathcal{H}_{1}$ associated with a function $\varphi \in G_{2}(\mathcal{F})$ such that $0<\lambda^{2}=\int_{I^{d}} \varphi^{2}(\mathbf{u}) \mathrm{d} \mathbf{u}<1$,

$$
\operatorname{dim} L\left(\Theta_{\varphi}\right) \geq d\left(1-\int_{I^{d}} \varphi^{2}(\mathbf{u}) \mathrm{d} \mathbf{u}\right)
$$

First we will discuss some consequences of this property. Let $\Lambda \in(0,1)$. By choosing $\lambda=\Lambda \in(0,1)$ and taking $\varphi(\mathbf{u})=\Lambda$ for $\mathbf{u} \in I^{d}$, the obvious inclusion $L\left(\Theta_{\varphi}\right) \subseteq L_{\Lambda}$ implies that

$$
\operatorname{dim} L_{\Lambda} \geq d\left(1-\Lambda^{2}\right) \text { for each } \Lambda \in(0,1) .
$$

We next suppose that (2.27) holds. It is obvious that $\operatorname{dim} L_{1} \geq 0$ and according to the properties of the Hausdorff dimension,

$$
\operatorname{dim} L_{0}=\operatorname{dim} \bigcup_{m \geq 1} L_{1 / m}=\sup _{m \geq 1} \operatorname{dim} L_{1 / m}=\sup _{m \geq 1}\left(1-m^{-2}\right)=1 .
$$

Thus, to prove that (1.11) holds for each $\Lambda \in[0,1]$, it is enough to efstablish $(2.26)$, for all $0<\lambda<1$.

For this purpose, we will apply Fact 2.2, taking for $A$ a suitable subset of $L\left(\Theta_{\varphi}\right)$ and $c=d\left(1-\lambda^{2}-\eta\right)$ with $\eta>0$ small enough. In the following, our attention will be devoted to the construction of $A$. We will require some additional notation. Let $\left\{h_{k}: k \geq 1\right\}$ be a sequence of constants verifying

H1) $h_{k} \rightarrow 0, k h_{k}^{d} \rightarrow \infty$ and $0<h_{k}<1$

$\mathrm{H} 2$ ) for all $0<c<1, \sum_{k=1}^{\infty} h_{k}^{-1} \exp \left\{-h_{k}^{-c} / 2\right\}$ is a convergent series.

For all $k \geq 1$, we set $m_{k}=\left\lfloor\left(h_{k}^{1 / d}\right)^{-1}\right\rfloor$ and $t_{k}(\mathbf{i})=\left(t_{k}\left(i_{1}\right), \ldots, t_{k}\left(i_{d}\right)\right)=\left(i_{1} h_{k}^{1 / d}, \ldots, i_{d} h_{k}^{1 / d}\right)$. We define for all $\varepsilon>0, \varphi \in G_{2}(\mathcal{F})$ and $k \geq 1$,

$$
\begin{gathered}
W_{k, \varphi}(\varepsilon)=\left\{\left(t_{k}\left(i_{1}\right), \ldots, t_{k}\left(i_{d}\right)\right): 1 \leq i_{1}, \ldots, i_{d} \leq m_{k},\right. \\
\left.\left\|\Theta_{h_{k}, t_{k}(\mathbf{i})}-\Theta_{\varphi}\right\|_{\mathcal{F}}<\varepsilon\right\}
\end{gathered}
$$

and

$$
U_{k, \varphi}(\varepsilon)=\left\{\mathbf{t} \in[0,1 / 2]^{d}:\left\|\Theta_{h_{k}, \mathbf{t}}-\Theta_{\varphi}\right\|_{\mathcal{F}}<\varepsilon\right\}
$$

The $\varepsilon$-neighborhood of a measurable set $V \subseteq I^{d}$, for $\varepsilon>0$, is defined by

$$
\mathbb{N}(\varepsilon, V)=\bigcup_{\mathbf{x}=\left(x_{1}, \ldots, x_{d}\right) \in V} \prod_{r=1}^{d}\left(x_{r}-\varepsilon, x_{r}+\varepsilon\right)
$$

For any $\mathbf{t} \in I^{d}$, we can choose $\mathbf{i}$ in order to minimize $\left|t_{k}(\mathbf{i})-\mathbf{t}\right|$. Before the construction of $A$, we show a few lemmas.

Lemma 2.7. For any $\varepsilon \in(0,1)$ and $\theta=\theta(\varepsilon)<1$ satisfying $\psi(\mathrm{d} \theta) \leq \varepsilon$, there exists almost surely a $k_{0}(\varepsilon, \theta)$ such that, for all $k \geq k_{0}(\varepsilon, \theta)$,

$$
\mathbb{N}\left(\theta h_{k}, W_{k, \varphi}(\varepsilon)\right) \subseteq U_{k, \varphi}(2 \varepsilon)
$$

Proof. Let $t_{k}(\mathbf{i})=\left(t_{k}\left(i_{1}\right), \ldots, t_{k}\left(i_{d}\right)\right) \in W_{k, \varphi}(\varepsilon)$ and $\mathbf{t}=\left(t_{1}, \ldots t_{d}\right) \in[0,1 / 2]^{d}$ be such that for each $1 \leq r \leq d$, we have $\left|t_{k}\left(i_{r}\right)-t_{r}\right|<\theta h_{k}^{1 / d}$. The triangle inequality entails that

$$
\left\|\Theta_{h_{k}, \mathbf{t}}-\Theta_{\varphi}\right\|_{\mathcal{F}} \leq\left\|\Theta_{h_{k}, \mathbf{t}}-\Theta_{h_{k}, t_{k}(\mathbf{i})}\right\|_{\mathcal{F}}+\left\|\Theta_{h_{k}, t_{k}(\mathbf{i})}-\Theta_{\varphi}\right\|_{\mathcal{F}}
$$


Using Lemmas 2.4 and 2.5, we conclude that for any $\varepsilon>0$, there exists $k_{0}(\varepsilon)$ such that for every $k \geq k_{0}(\varepsilon)$

$$
\left\|\Theta_{h_{k}, \mathbf{t}}-\Theta_{h_{k}, t_{k}(\mathbf{i})}\right\|_{\mathcal{F}}<\psi(\mathrm{d} \theta) .
$$

So,

$$
\left\|\Theta_{h_{k}, \mathbf{t}}-\Theta_{\varphi}\right\|_{\mathcal{F}} \leq 2 \varepsilon
$$

The conclusion of the lemma is therefore immediate.

For every measurable set $E \subseteq I^{d}$, we set

$$
\begin{aligned}
& m_{k}(E)=\#\left\{\left(i_{1}, \ldots, i_{d}\right): 0 \leq i_{1}, \ldots, i_{d} \leq m_{k},\right. \\
&\left.\prod_{r=1}^{d}\left[t_{k}\left(i_{r}\right), t_{k}\left(i_{r}+1\right)\right] \subseteq E\right\},
\end{aligned}
$$

and, remembering (1.6)

$$
\begin{aligned}
N_{k, \varphi}(\varepsilon)=\#\left\{\left(i_{1}, \ldots, i_{d}\right): 1 \leq\right. & i_{1}, \ldots, i_{d} \leq m_{k}, \\
& \left.\left\|\Theta_{h_{k}, t_{k}(\mathbf{i})}-\Theta_{\varphi}\right\|_{\mathcal{F}} \leq \varepsilon\right\} .
\end{aligned}
$$

For every measurable set $E \subseteq I^{d}$ we define $N_{k, \varphi}(\varepsilon, E)$ to be

$$
\begin{aligned}
N_{k, \varphi}(\varepsilon ; E)=\#\{ & \left(i_{1}, \ldots, i_{d}\right): 1 \leq i_{1}, \ldots, i_{d} \leq m_{k} ; \\
& \left.\left(t_{k}\left(i_{1}\right), \ldots, t_{k}\left(i_{d}\right)\right) \in E,\left\|\Theta_{h_{k}, t_{k}(\mathbf{i})}-\Theta_{\varphi}\right\|_{\mathcal{F}}<\varepsilon\right\} .
\end{aligned}
$$

Finally, for every $0 \leq i_{1}, \ldots, i_{d} \leq m_{k}$, we set

$$
X_{i_{1}, \ldots, i_{d}}=\mathbb{1}\left\{\left\|\Theta_{h_{k}, t_{k}(\mathbf{i})}-\Theta_{\varphi}\right\|_{\mathcal{F}}<\varepsilon\right\} .
$$

Observe that these variables are independent and identically distributed Bernouilli random variables with probability of success

$$
p_{k}(\varepsilon):=P\left(X_{\mathbf{0}}=1\right)=P\left(\left\|\Theta_{h_{k}, \mathbf{0}}-\Theta_{\varphi}\right\|_{\mathcal{F}}<\varepsilon\right) .
$$

The following lemma provides an evaluation of this probability.

Lemma 2.8. For all $\delta \in\left(0, \lambda^{2}\right)$, there exist $0<\delta^{\prime}<\delta, \varepsilon_{0}=\varepsilon_{0}(\delta)>0$ and a $k_{1}(\varepsilon, \delta) \geq 1$ such that for each $\varepsilon \in\left(0, \varepsilon_{0}\right]$ and $k \geq k_{1}(\varepsilon, \delta)$, we have

$$
p_{k}(\varepsilon) \geq h_{k}^{\lambda^{2}-\delta^{\prime}} \geq h_{k}^{\lambda^{2}}
$$

Proof. We set

$$
N_{\varepsilon}\left(\Theta_{\varphi}\right)=\left\{\Xi \in \ell_{\infty}(\mathcal{F}):\left\|\Xi-\Theta_{\varphi}\right\|_{\mathcal{F}}<\varepsilon\right\} .
$$

Recall the definition (1.6) of $\Theta_{h_{k}, t_{k}(\mathbf{i})}$ and (2.36). Observe that

$$
p_{k}(\varepsilon)=P\left(\Theta_{h_{k}, \mathbf{0}} \in N_{\varepsilon}\left(\Theta_{\varphi}\right)\right) .
$$

Setting $G=N_{\varepsilon}\left(\Theta_{\varphi}\right)$, which is an open set of $\ell_{\infty}(\mathcal{F})$, we apply Fact 2.1 (ii), with $\varpi_{k}=h_{k}$. We obtain then for all $k$ large enough,

$$
p_{k}(\varepsilon) \geq \exp \left\{-\varepsilon_{k}^{-1} I\left(N_{\varepsilon}\left(\Theta_{\varphi}\right)\right)\right\} \geq \exp \left\{-2 \log \left(h_{k}^{-1}\right) I\left(N_{\varepsilon}\left(\Theta_{\varphi}\right)\right)\right\} .
$$

But, $I\left(N_{\varepsilon}\left(\Theta_{\varphi}\right)\right)<I\left(\Theta_{\varphi}\right)=\lambda^{2} / 2$. Thus, for any fixed $\delta>0$, there exists an $\varepsilon_{0}(\delta)>0$ such that for each $\varepsilon \in\left(0, \varepsilon_{0}(\delta)\right], \lambda^{2}-\delta<2 I\left(N_{\varepsilon}\left(\Theta_{\varphi}\right)\right)<\lambda^{2}$. It follows that (2.37) holds choosing $\delta^{\prime} \in\left(0, \lambda^{2}-2 I\left(N_{\varepsilon}\left(\Theta_{\varphi}\right)\right)\right)$. 
Let $E$ be an union of disjoint hypercubes of Lebesgue measure $\operatorname{vol}(E)$ greater than $S \geq 3^{d} h_{k}$. Then we have

$$
\frac{\operatorname{vol}(E)}{3^{d} h_{k}} \leq\left(1-\frac{2 h_{k}^{1 / d}}{S^{1 / d}}\right)^{d} \frac{\operatorname{vol}(E)}{h_{k}} \leq m_{k}(E) \leq \frac{\operatorname{vol}(E)}{h_{k}} .
$$

Lemma 2.9. Let $\varepsilon \in\left(0, \varepsilon_{0}\right)$ where $\varepsilon_{0}$ is as in Lemma 2.8 and let $\delta \in\left(0,1-\lambda^{2}\right)$ be fixed. For any $\sigma \in(0,1)$ there exists almost surely a $k_{2}(\varepsilon, \sigma, \delta) \geq 1$ such that, for all $k \geq k_{2}(\varepsilon, \sigma, \delta)$, we have

$$
\left|N_{k, \varphi}(\varepsilon, E)-m_{k}(E) p_{k}(\varepsilon)\right|<\sigma m_{k}(E) p_{k}(\varepsilon)
$$

where $E$ is an union of disjoint hypercubes of Lebesgue measure $\operatorname{vol}(E)$ greater than $h_{k}^{1-\lambda^{2}-\delta}$

Proof. Since $N_{k, \varphi}(\varepsilon,$.$) and m_{k}($.$) are additive set functions, it suffices to prove (2.40) when E$ is a hypercube of $I^{d}$ with Lebesgue measure greater than $h_{k}^{1-\lambda^{2}-\delta}$.

Fix $\sigma^{\prime}$ and $\delta^{\prime}$ such that $0<\sigma^{\prime}<\sigma$ and $0<\delta^{\prime}<\delta$. We next prove that it is enough to prove the lemma when $E=J$ is a hypercube of the form

$$
\prod_{r=1}^{d}\left[t_{k}\left(i_{r}\right), t_{k}\left(i_{r}+l(k)\right)\right)
$$

for some $0 \leq i_{1}, \ldots, i_{d} \leq 2 m_{k}$, and with $l(k):=\left\lfloor h_{k}^{-\left(\lambda^{2}+\delta^{\prime}\right) / d}\right\rfloor$. To see this, assume that (2.40) is satisfied for $E=J, \sigma=\sigma^{\prime}$ and $\delta=\delta^{\prime}$. Let $K(k):=\left\lfloor h_{k}^{\delta^{\prime}-\delta}\right\rfloor$. From (H1), we can choose $k(\delta, \sigma)<\infty$ such that, for all $k \geq k(\delta, \sigma)$, we have

$$
\begin{gathered}
3 \leq K(k), \\
3^{d} h_{k}<h_{k}^{1-\lambda^{2}-\delta}, \\
\left(1+\sigma^{\prime}\right)\left\{\frac{1+4 /(K(k)-2)}{1-2 h_{k}^{\left(\lambda^{2}+\delta\right) / d}}\right\}^{d} \leq(1+\sigma),
\end{gathered}
$$

and

$$
1-\sigma \leq\left(1-\sigma^{\prime}\right)\left\{\frac{1-2 h_{k}^{\left(\lambda^{2}+\delta^{\prime}\right) / d}}{1+4 /(K(k)-2)}\right\}^{d} .
$$

For all $k \geq 1$, we have

$$
K:=K(E, k)=\left\lfloor\frac{\operatorname{vol}(E)^{1 / d}}{h_{k}^{\left(1-\lambda^{2}-\delta^{\prime}\right) / d}}\right\rfloor .
$$

Observe that $K \geq K(k) \rightarrow \infty$. For $k \geq k(\delta, \sigma)$, there exist $(K+2)^{d}$ disjoint hypercubes $J_{1}, \ldots, J_{(K+2)^{d}}$ of the form $\prod_{r=1}^{d}\left[t_{k}\left(i_{r}\right), t_{k}\left(i_{r}+l(k)\right)\right]$ such that

$$
\bigcup_{\ell=1}^{(K-2)^{d}} J_{\ell} \subseteq E \subseteq \bigcup_{\ell=1}^{(K+2)^{d}} J_{\ell}
$$

Given the form of the hypercubes $J_{\ell}$, we see that $\operatorname{vol}\left(J_{\ell}\right)=(1+o(1)) h_{k}^{1-\lambda^{2}-\delta^{\prime}}$. Moreover, we have the following inequalities.

$$
(K-2)^{d} \operatorname{vol}\left(J_{1}\right) \leq \operatorname{vol}(E) \leq(K+2)^{d} \operatorname{vol}\left(J_{1}\right) .
$$


Hence, applying (2.39) and Lemma 2.9 for $E=J_{\ell}$, it follows that, for all $k$ large enough,

$$
\begin{aligned}
N_{k, \varphi}(\varepsilon, E) & \leq \sum_{\ell=1}^{(K+2)^{d}} N_{k, \varphi}\left(\varepsilon, J_{\ell}\right) \leq\left(1+\sigma^{\prime}\right)\left\{\sum_{\ell=1}^{(K+2)^{d}} m_{k}\left(J_{\ell}\right)\right\} p_{k}(\varepsilon) \\
& \leq(K+2)^{d}\left(1+\sigma^{\prime}\right) \operatorname{vol}\left(J_{1}\right) h_{k}^{-1} p_{k}(\varepsilon) \\
& \leq \frac{(K+2)^{d}}{(K-2)^{d}}\left(1+\sigma^{\prime}\right) \operatorname{vol}(E) h_{k}^{-1} p_{k}(\varepsilon) \\
& \leq\left(1+\sigma^{\prime}\right)\left\{\frac{1+\frac{4}{K-2}}{1-2 h_{k}^{\left(\lambda^{2}+\delta\right) / d}}\right\}^{d} m_{k}(E) p_{k}(\varepsilon) \\
& \leq(1+\sigma) m_{k}(E) p_{k}(\varepsilon) .
\end{aligned}
$$

A similar argument shows that

$$
\begin{aligned}
N_{k, \varphi}(\varepsilon, E) & \geq \sum_{\ell=1}^{(K-2)^{d}} N_{k, \varphi}\left(\varepsilon, J_{\ell}\right) \geq(K-2)^{d} N_{k}\left(J_{1}\right) \\
& \geq(K-2)^{d}\left(1-\sigma^{\prime}\right) m_{k}\left(J_{1}\right) p_{k}(\varepsilon) \\
& \geq(K-2)^{d}\left(1-\sigma^{\prime}\right) \frac{\operatorname{vol}\left(J_{1}\right)}{h_{k}}\left(1-2 h_{k}^{\left(\Lambda_{1}^{2}+\delta^{\prime}\right) / d}\right)^{d} p_{k}(\varepsilon) \\
& \geq \frac{(K-2)^{d}}{(K+2)^{d}}\left(1-\sigma^{\prime}\right) \frac{\operatorname{vol}(E)}{h_{k}}\left(1-2 h_{k}^{\left(\Lambda_{1}^{2}+\delta^{\prime}\right) / d}\right)^{d} p_{k}(\varepsilon) \\
& \geq\left(1-\sigma^{\prime}\right)\left\{\frac{1-2 h_{k}^{\left(\lambda^{2}+\delta^{\prime}\right) / d}}{1+\frac{4}{K-2}}\right\}^{d} m_{k}(E) p_{k}(\varepsilon) \\
& \geq(1-\sigma) m_{k}(E) p_{k}(\varepsilon) .
\end{aligned}
$$

We obtain that it suffices to show the lemma when $E$ is a hypercube $J$ of the form

$$
J=\prod_{r=1}^{d}\left[t_{k}\left(i_{r}\right), t_{k}\left(i_{r}+\ell(k)\right)\right] \text { with } 0 \leq i_{r} \leq 2 m_{k} \text { and } 1 \leq r \leq d .
$$

Note here that the total number of hypercubes of this form is bounded above by $h_{k}^{-1}$. For all $k \geq 1$, we set $Q_{k}=P\left(N_{k, \varphi}(\varepsilon, J)>r_{k, 1}\right)$ where $r_{k, 1}=\left(1+\sigma^{\prime}\right) m_{k}(J) p_{k}(\varepsilon)$. We now apply Fact 2.3 for $S_{N}=N_{k, \varphi}(\varepsilon, J)$, $N=m_{k}(J), p=p_{k}(\varepsilon)$ and $r=\left(1+\sigma^{\prime}\right)$. We obtain for $k$ sufficiently large,

$$
Q_{k} \leq \exp \left\{-m_{k}(J) p_{k}(\varepsilon) h\left(1+\sigma^{\prime}\right)\right\} .
$$

Moreover, for $k$ large enough, we have

$$
m_{k}(J) \geq\left[\frac{\operatorname{vol}(J)^{1 / d}}{h_{k}^{1 / d}}-2\right]^{d} \geq \frac{1}{2^{d}} h_{k}^{-\lambda^{2}-\delta^{\prime}} .
$$

Thus, applying Lemma 2.8, we see that for $k$ large enough,

$$
h_{k}^{-1} Q_{k} \leq h_{k}^{-1} \exp \left\{-\frac{1}{2^{d}} h_{k}^{-\delta^{\prime}} h\left(1+\sigma^{\prime}\right)\right\} .
$$


We use (H2) to obtain $\sum_{k=1}^{\infty} h_{k}^{-1} Q_{k}<\infty$. Therefore, the Borel-Cantelli lemma implies that with probability 1 , for all $k$ sufficiently large, we have

$$
N_{k, \varphi}(\varepsilon, J) \leq\left(1+\sigma^{\prime}\right) m_{k}(J) p_{k}(\varepsilon) .
$$

Define likewise $R_{k}=P\left(N_{k, \varphi}(\varepsilon, J)<r_{k, 2}\right)$ where $r_{k, 2}=\left(1-\sigma^{\prime}\right) m_{k}(J) p_{k}(\varepsilon)$. By a similar argument, we show that with probability 1 , for all $k$ sufficiently large,

$$
N_{k, \varphi}(\varepsilon, J) \geq\left(1-\sigma^{\prime}\right) m_{k}(J) p_{k}(\varepsilon) .
$$

Finally, (2.40) is proved for any hypercube of the form (2.47). This concludes the proof of Lemma 2.9.

We shall now prove the existence of a sequence of sets $E_{1}, E_{2}, \ldots$ fulfilling the assumptions of Fact 2.2 and such that $A=\bigcap_{m=1}^{\infty} E_{m} \subseteq L\left(\Theta_{\varphi}\right)$. Let see the steps needed for the construction of these sets. In a first step, we establish the existence of this sequence via an induction argument. In a second step, we show that $\left\{E_{m}: m \geq 1\right\}$ satisfies (2.5). Finally, in a last step, we apply the Fact 2.2 to establish (2.2).

SteP 1 : Existence of $E_{m}$. We choose two sequences of nonnegative constants $\left\{\sigma_{m}: m \geq 1\right\}$ and $\left\{\delta_{m}: m \geq 1\right\}$ such that

(1i) $0<\sigma_{m}<1 / 2$, for any $m \geq 1$;

(1ii) $\prod_{i=1}^{m}\left(1+\sigma_{i}\right)^{2} /\left(1-\sigma_{i}\right)^{2} \leq 2$;

(1iii) $\delta_{0}=0, \delta_{m} \geq 0$, for any $m \geq 1$;

(1iv) $\sum_{m=1}^{\infty} \delta_{m} \leq \eta / 3<\frac{1}{6} \min \left(\lambda^{2}, 1-\lambda^{2}\right)$.

We select two decreasing sequences of positive constants $\left\{\varepsilon_{m}: m \geq 1\right\}$ and $\left\{\theta_{m}: m \geq 1\right\}$ such that

(2i) $\varepsilon_{1}<1, \varepsilon_{m} \downarrow 0, \varepsilon_{m}<\delta_{m}$;

(2ii) $\theta_{m}<(1 / 2) \varepsilon_{m}^{2}$;

(2iii) $\left(1+2 \theta_{m-1}\right)^{d\left(1-\lambda^{2}-2 \Delta_{m-1}\right)} \leq \frac{1+\sigma_{m}}{1+1 / 2 \sigma_{m}}$;

(2iv) $3^{d\left(1-\lambda^{2}-2 \Delta_{m-1}\right)} \leq \frac{1+\sigma_{m}}{1+1 / 2 \sigma_{m}}$.

For further use, we choose $k_{2}\left(\varepsilon_{m}, \sigma_{m}, \delta_{m}\right) \geq k_{1}\left(\varepsilon_{m}, \delta_{m} \geq k_{0}\left(\varepsilon_{m}\right)\right.$ as in Lemmas 2.7, 2.8 and 2.9. We set $\Delta_{m}=\sum_{k=1}^{m} \delta_{k}$. Observe that for every $m \geq 1$,

$$
\Delta_{m}<\frac{1}{6} \min \left(\lambda^{2}, 1-\lambda^{2}\right),
$$

and $0<\delta_{m}<\Delta_{m}<1$. The construction of the sets $E_{m}$ relies on an inductive argument. That is to say that given $E_{m-1}$ and $\left\{M_{m-1}, k_{m-1}, E_{m-1}^{*}\right\}$, we evaluate $E_{m}$ and $\left\{M_{m}, k_{m}, E_{m}^{*}\right\}$. The constants $M_{m-1}, k_{m-1}$ and the sets $E_{m-1}^{*}$ are defined below.

We set

and, for every $m \geq 1$,

$$
k_{0}=1, \quad L_{0}=1, \quad M_{0}=1,
$$

$$
L_{m}=\theta_{m} h_{k_{m}}^{1 / d} \quad \text { and } \quad L_{m-1}^{*}=L_{m-1}-L_{m}
$$

We choose $k_{m}$ a positive integer such that the following conditions are verified.

(3i) $k_{m}>\max \left\{k_{m-1}, k_{2}\left(\varepsilon_{m}, \sigma_{m}, \delta_{m}\right)\right\}$;

(3ii) $3^{d} L_{m}<3^{d} h_{k_{m}}^{1 / d}<h_{k_{m}}^{\left(1-\lambda^{2}-\delta_{m}\right) / d}<L_{m-1}^{*}<L_{m-1}$;

(3iii) $L_{m-1}^{d} /\left(L_{m-1}^{*}\right)^{d} \leq 1+(1 / 2) \sigma_{m}$;

(3iv) $1-\sigma_{m} \leq\left(1-\frac{2 h_{k_{m}}^{1 / d}}{L_{m-1}^{*}}\right)^{d}$;

(3v) $2 \frac{h_{k_{m}}^{\delta_{m}}}{h_{k_{m}-1}^{\lambda^{2} \delta_{m-1}}} \leq \theta_{m}^{d}$ for $m \geq 2$; 
(3vi) $2\left(1-3^{-d}\right)^{-d} h_{k_{m}}^{\delta_{m}} \leq \theta_{m}^{d}$, for any $m \geq 1$

(3vii) $6 h_{k_{m}}^{\delta_{m}} \leq \theta_{m}^{d} M_{m-1}\left(L_{m-1}^{*}\right)^{d}$, for $m \geq 1$.

By (H1), we can choose $k_{m}$ such that (3i-3vii) hold. Now, for every $m \geq 1$, given $k_{m}$ and $\left\{M_{m-1}, k_{m-1}, E_{m-1}^{*}\right\}$, we define the sets $E_{m}, E_{m}^{*}$, and the positive integer $M_{m}$. We set

$$
E_{m}=\underset{\substack{1 \leq i_{1}, \ldots, i_{r} \leq m_{k_{m}} \\\left(t_{k_{m}}\left(i_{1}\right), \ldots, t_{k_{m}}\left(i_{d}\right)\right) \in W_{k_{m}, \varphi}\left(\varepsilon_{m}\right) \cap E_{m-1}^{*}}}{\bigcup_{r=1}^{d}} \prod_{r}\left[t_{k_{m}}\left(i_{r}\right), t_{k_{m}}\left(i_{r}\right)+L_{m}\right]
$$

with $E_{0}=I^{d}$

$$
\left.E_{m}^{*}=\underset{\substack{1 \leq i_{1}, \ldots, i_{r} \leq m_{k_{m}} \\\left(t_{k_{m}}\left(i_{1}\right), \ldots, t_{k_{m}}\left(i_{d}\right)\right) \in W_{k_{m}, \varphi}\left(\varepsilon_{m}\right) \cap E_{m-1}^{*}}}{\bigcup_{r=1}^{d}} \prod_{t_{m}}^{d}\left(t_{k_{m}}\right), t_{k_{m}}\left(i_{r}\right)+L_{m}^{*}\right]
$$

with $E_{0}=\left[0, L_{0}^{*}\right]^{d}$. Recalling $(2.34)$, we set $M_{0}=1$ and for all $m \geq 1$

$$
\begin{aligned}
M_{m}:=N_{k_{m}, \varphi}\left(\varepsilon_{m}, E_{m-1}^{*}\right)=\# & \left\{\left(i_{1}, \ldots, i_{d}\right): i_{1}, \ldots, i_{d}=1, \ldots, m_{k_{m}},\right. \\
& \left.\left(t_{k_{m}}\left(i_{1}\right), \ldots, t_{k_{m}}\left(i_{d}\right)\right) \in W_{k_{m}, \varphi}\left(\varepsilon_{m}\right) \cap E_{m-1}^{*}\right\} .
\end{aligned}
$$

It is obvious that for all $m \geq 0, E_{m}$ (resp. $E_{m}^{*}$ ) is an union of $M_{m}$ hypercubes of Lebesgue measure $L_{m}$ (resp. $L_{m}^{*}$ ), which will be denoted by $J_{m, \ell}\left(\operatorname{resp} . J_{m, \ell}^{*}\right)$ for $\ell=1, \ldots, M_{m}$. Moreover, these hypercubes are disjoint since $t_{k_{m}}\left(i_{r}\right)+L_{m}<t_{k_{m}}\left(i_{r}+1\right)$ and $t_{k_{m}}\left(i_{r}\right)+L_{m}^{*}<t_{k_{m}}\left(i_{r}+1\right)$ by $(3 \mathrm{ii})$. We have for $m \geq 0$

$$
E_{m}=\bigcup_{\ell=1}^{M_{m}} J_{m, \ell} \text { and } E_{m}^{*}=\bigcup_{\ell=1}^{M_{m}} J_{m, \ell}^{*} .
$$

By construction,

$$
E_{m} \subseteq E_{m-1} \quad \text { and } \quad E_{m}^{*} \subseteq E_{m-1}^{*} .
$$

To prove that the induction process involved in the construction of the sets $E_{m}$ carries from stage $m-1$ to stage $m$, we need to verify that $M_{m} \geq 1$ for any $m \geq 1$. This is shown with the help of the lemma below.

Lemma 2.10. We have $M_{0}\left(L_{0}^{*}\right)^{d} \geq\left(1-3^{-d}\right)^{d}$. Moreover, if $M_{m-1} \geq 1$ for $m \geq 1$, then

$$
M_{m}\left(L_{m}^{*}\right)^{d} \geq h_{k_{m}}^{\lambda^{2}+\delta_{m}}
$$

Proof. (2.48) and (3ii) imply that

$$
M_{0}\left(L_{0}^{*}\right)^{d}=\left(L_{0}^{*}\right)^{d}=\left(L_{0}-L_{1}\right)^{d} \geq\left(L_{0}-3^{-d} L_{0}^{*}\right)^{d} \geq\left(1-3^{-d}\right)^{d} .
$$

Assume that $\left\{M_{k}, h_{k}, E_{k}, E_{k}^{*}\right\}$ have been defined for all $k \in\{0, \ldots, m-1\}$, and that $M_{m-1} \geq 1$. We apply Lemma 2.9 for $\delta=\delta_{m}, \sigma=\sigma_{m}, E=E_{m-1}^{*} \cdot \operatorname{vol}\left(E_{m-1}^{*}\right)=M_{m-1}\left(L_{m-1}^{*}\right)^{d} \geq\left(L_{m-1}^{*}\right)^{d} \geq h_{k_{m}}^{1-\lambda^{2}-\delta_{m}}$ using $(3 \mathrm{ii})$. Recalling (2.52), one obtains :

$$
1-\sigma_{m} \leq \frac{M_{m}}{m_{k_{m}}\left(E_{m-1}^{*}\right) p_{k_{m}}(\varepsilon)} \leq 1+\sigma_{m} .
$$

We may also apply $(2.39)$ with $k=k_{m}$ and $S=\left(L_{m-1}^{*}\right)^{d}$, to have

$$
\left(1-\frac{2 h_{k_{m}}^{1 / d}}{L_{m-1}^{*}}\right)^{d} \frac{\operatorname{vol}\left(E_{m-1}^{*}\right)}{h_{k_{m}}} \leq m_{k_{m}}\left(E_{m-1}^{*}\right) \leq \frac{\operatorname{vol}\left(E_{m-1}^{*}\right)}{h_{k_{m}}}
$$


By (3iv), we see that

$$
\left(1-\sigma_{m}\right) h_{k_{m}}^{-1} \leq \frac{m_{k_{m}}\left(E_{m-1}^{*}\right)}{M_{m-1}\left(L_{m-1}^{*}\right)^{d}} \leq h_{k_{m}}^{-1} .
$$

Using now Lemma 2.8, for $k=k_{m}, \delta^{\prime}=\delta_{m}$ and $\varepsilon=\varepsilon_{m}$, we get

$$
h_{k_{m}}^{\lambda^{2}} \leq h_{k_{m}}^{\lambda^{2}-\delta_{m}} \leq p_{k_{m}}\left(\varepsilon_{m}\right)
$$

By (2.56) and (2.58), we have

$$
\begin{aligned}
\left(1-\sigma_{m}\right)^{2} h_{k_{m}}^{-1+\lambda^{2}} & \leq\left(1-\sigma_{m}\right)^{2} h_{k_{m}}^{-1} p_{k_{m}}\left(\varepsilon_{m}\right) \\
& \leq \frac{M_{m}}{m_{k_{m}}\left(E_{m-1}^{*}\right) p_{k_{m}}\left(\varepsilon_{m}\right)} \frac{m_{k_{m}}\left(E_{m-1}^{*}\right)}{M_{m-1}\left(L_{m-1}^{*}\right)^{d}} p_{k_{m}}\left(\varepsilon_{m}\right) \\
& \leq \frac{M_{m}}{M_{m-1}\left(L_{m-1}^{*}\right)^{d}}
\end{aligned}
$$

By (1i), notice that for all $m \geq 1$, the inequalities $1 / 2 \leq 1-\sigma_{m+1}$ and $1+\sigma_{m+1} \leq 3 / 2$ hold. Then, we get

$$
1 / 6 \leq\left(1-\sigma_{m}\right)^{2} \frac{1}{1+\sigma_{m+1}} .
$$

These inequalities when combined with (3iii) imply that

$$
\begin{aligned}
\frac{1}{6} \theta_{m}^{d} M_{m-1}\left(L_{m-1}^{*}\right)^{d} h_{k_{m}}^{\lambda^{2}} & \leq \frac{\left(1-\sigma_{m}\right)^{2}}{\left(1+\sigma_{m+1}\right)} M_{m-1}\left(L_{m-1}^{*}\right)^{d} h_{k_{m}}^{-1+\lambda^{2}} h_{k_{m}} \theta_{m}^{d} \\
& \leq M_{m} \frac{1}{1+\sigma_{m+1}} L_{m}^{d} \leq M_{m}\left(L_{m}^{*}\right)^{d}
\end{aligned}
$$

We infer from (3vii) that

$$
M_{m}\left(L_{m}^{*}\right)^{d} \geq h_{k_{m}}^{\lambda^{2}+\delta_{m}}
$$

This completes the proof of Lemma 2.10.

We now apply Lemma 2.10, to prove that $M_{m} \geq 1$ for all $m \geq 1$. By (1iv), we easily see that $\lambda^{2}-1+\delta_{m} \leq$ $-2 / 3\left(1-\lambda^{2}\right)<0$. Next, by $(2 \mathrm{ii}), \theta_{m}<(1 / 2) \varepsilon_{m}^{2}<1 / 2$. So, $L_{m}^{*}<L_{m}<h_{k_{m}}^{1 / d} / 2$. We use Lemma 2.10 to see that $M_{m} \geq 2^{d} h_{k_{m}}^{-1+\lambda^{2}+\delta_{m}} \geq 2^{d} h_{k_{m}}^{-2\left(1-\lambda^{2}\right) / 3}$. This last inequality, combined with (H1) and $h_{k_{m}}<1$ jointly imply that $M_{m} \geq 2^{d} \geq 1$.

This last property establishes the existence of $\left\{M_{m}, k_{m}, E_{m}, E_{m}^{*}\right\}$, for all $m \geq 0$.

STEP $2:$ Properties of $E_{m}$. In this step, we prove the existence of the constants $c, d$, and $\Delta$ such that the inequality (2.5) of Fact 2.2 is verified for any hypercube $J \subseteq I^{d}$ with $|J| \leq \Delta$. We see, remembering (2.5) and (2.34), that for any hypercube $J \subseteq I^{d}$ and for $m \geq 1$

$$
M_{m}(J) \leq N_{k_{m}, \varphi}\left(\varepsilon_{m}, J\right) .
$$

We have to distinguish several cases, depending on the nature of the hypercube $J \subseteq I^{d}$.

Case 1.: for some $m \geq 1, J \subseteq J_{m-1, \ell_{0}}$, for some $\ell_{0} \in\left\{1, \ldots, M_{m-1}\right\}$,

Case 2.: for some $m \geq 1, J \cap J_{m-1, \ell_{0}} \neq \varnothing$, for some $\ell_{0} \in\left\{1, \ldots, M_{m-1}\right\}$,

Case 3.: for some $m \geq 1, J \cap J_{m-1, \ell}=\varnothing, \forall \ell \in\left\{1, \ldots, M_{m-1}\right\}$. 
Case 1. For $m \geq 1$, assume that $J \subseteq J_{m-1, \ell_{0}}$ where $\ell_{0} \in\left\{1, \ldots, M_{m-1}\right\}$. Three possibilities have to be considered.

Possibility 1a. Assume that $\operatorname{vol}(J) \geq h_{k_{m}}^{1-\lambda-\delta_{m}}$ (This is possible by (3ii)). We may apply Lemma 2.9, with $\delta=\delta_{m}, \sigma=\sigma_{m}, E=J$, and $k=k_{m}$, combined with (2.39) and (2.64), to obtain

$$
M_{m}(J) \leq\left(1+\sigma_{m}\right) h_{k_{m}}^{-1} \operatorname{vol}(J) p_{k_{m}}\left(\varepsilon_{m}\right)
$$

When $m \geq 2$, we have

$$
\operatorname{vol}(J) \leq \operatorname{vol}\left(J_{m-1, k_{0}}\right)=L_{m-1}^{d}=\theta_{m-1}^{d} h_{k_{m-1}} \leq h_{k_{m-1}} .
$$

Then, using Lemma 2.10, we get

$$
M_{m-1}\left(L_{m-1}^{*}\right)^{d} \geq h_{k_{m-1}}^{\lambda^{2}+\delta_{m-1}} \geq \operatorname{vol}(J)^{\lambda^{2}+\delta_{m-1}} .
$$

Next, (2.59) and (2.60) jointly imply that

$$
\begin{aligned}
\frac{M_{m}(J)}{M_{m}} & =\frac{M_{m-1}\left(L_{m-1}^{*}\right)^{d}}{M_{m}} M_{m}(J) \frac{1}{M_{m-1}\left(L_{m-1}^{*}\right)^{d}} \\
& \leq \frac{1}{\left(1-\sigma_{m}\right)^{2} h_{k_{m}}^{-1} p_{k_{m}}\left(\varepsilon_{m}\right)}\left(1+\sigma_{m}\right) h_{k_{m}}^{-1} \operatorname{vol}(J) p_{k_{m}}\left(\varepsilon_{m}\right) \frac{1}{M_{m-1}\left(L_{m-1}^{*}\right)^{d}} \\
& \leq \frac{1+\sigma_{m}}{\left(1-\sigma_{m}\right)^{2}} \operatorname{vol}(J) \frac{1}{M_{m-1}\left(L_{m-1}^{*}\right)^{d}} \\
& \leq \frac{\left(1+\sigma_{m}\right)}{\left(1-\sigma_{m}\right)^{2}} \operatorname{vol}(J)^{1-\lambda^{2}-\delta_{m-1}} \\
& \leq \frac{\left(1+\sigma_{m}\right)}{\left(1-\sigma_{m}\right)^{2}} d^{-d / 2\left(1-\lambda^{2}-\delta_{m-1}\right)}|J|^{d-d \lambda^{2}-d \delta_{m-1}}
\end{aligned}
$$

where $|A|$ is the diameter of $A$ (see p.250). When $m=1$, we know that $M_{0}\left(L_{0}^{*}\right)^{d} \geq\left(1-3^{-d}\right)^{d}$, therefore,

$$
\begin{aligned}
\frac{M_{1}(J)}{M_{1}} & \leq\left(1-3^{-d}\right)^{-d} \frac{1+\sigma_{1}}{\left(1-\sigma_{1}\right)^{2}} \operatorname{vol}(J) \\
& \leq\left(1-3^{-d}\right)^{-d} \frac{1+\sigma_{1}}{\left(1-\sigma_{1}\right)^{2}} d^{-d / 2}|J|^{d} \\
& \leq\left(1-3^{-d}\right)^{-d} \frac{1+\sigma_{1}}{\left(1-\sigma_{1}\right)^{2}} d^{-d / 2}|J|^{d\left(1-\lambda^{2}\right)}
\end{aligned}
$$

Possibility 1b. Consider the possibility where

$$
\left(\theta_{m} h_{k_{m}}^{1 / d}\right)^{d} / 2 \leq \operatorname{vol}(J) \leq h_{k_{m}}^{1-\lambda^{2}-\delta_{m}}
$$

In this case, it is always possible to choose a hypercube $J^{\prime}$, such that $J \subseteq J^{\prime} \subseteq I^{d}$ with $\operatorname{vol}\left(J^{\prime}\right)=h_{k_{m}}^{1-\lambda^{2}-\delta_{m}}$. Then, it is easy to see that $h_{k_{m}}^{1 / d} \leq \theta_{m}^{-1}(2 \operatorname{vol}(J))^{1 / d}$ and therefore,

$$
h_{k_{m}}^{1-\lambda^{2}-2 \delta_{m}} \leq 2 \theta_{m}^{-d} \operatorname{vol}(J)^{1-\lambda^{2}-2 \delta_{m}}
$$


For any $m \geq 2$, apply (2.67) with the formal replacement of $J$ by $J^{\prime}$. This combined with (2.68) and (3v), implies

$$
\begin{aligned}
\frac{M_{m}(J)}{M_{m}} & \leq \frac{M_{m}\left(J^{\prime}\right)}{M_{m}} \leq \frac{1+\sigma_{m}}{\left(1-\sigma_{m}\right)^{2}} \operatorname{vol}\left(J^{\prime}\right) h_{k_{m-1}}^{-\lambda^{2}-\delta_{m-1}} \\
& \leq \frac{1+\sigma_{m}}{\left(1-\sigma_{m}\right)^{2}} h_{k_{m}}^{1-\lambda^{2}-\delta_{m}} h_{k_{m-1}}^{-\lambda^{2}-\delta_{m-1}} \\
& \leq \frac{1+\sigma_{m}}{\left(1-\sigma_{m}\right)^{2}} h_{k_{m}}^{1-\lambda^{2}-2 \delta_{m}} \frac{h_{k_{m}}^{\delta_{m}}}{h_{k_{m}-1}^{\lambda^{2} \delta_{m-1}}} \\
& \leq \frac{1+\sigma_{m}}{\left(1-\sigma_{m}\right)^{2}} \operatorname{vol}(J)^{1-\lambda^{2}-2 \delta_{m}} 2 \theta_{m}^{-d} \frac{h_{k_{m}}^{\delta_{m}}}{h_{k_{m-1}}^{\lambda^{2} \delta_{m-1}}} \\
& \leq \frac{1+\sigma_{m}}{\left(1-\sigma_{m}\right)^{2}} \operatorname{vol}(J)^{1-\lambda^{2}-2 \delta_{m}} \\
& \leq \frac{1+\sigma_{m}}{\left(1-\sigma_{m}\right)^{2}} d^{-d / 2\left(1-\lambda^{2}-2 \delta_{m}\right)}|J|^{d-d \lambda^{2}-2 d \delta_{m}} .
\end{aligned}
$$

For $m=1$, applying the same result, with the formal replacement of $J$ by $J^{\prime}$, and with (3vi), we obtain

$$
\begin{aligned}
\frac{M_{1}(J)}{M_{1}} & \leq\left(1-3^{-d}\right)^{-d} \frac{1+\sigma_{1}}{\left(1-\sigma_{1}\right)^{2}} \operatorname{vol}\left(J^{\prime}\right) \\
& \leq\left(1-3^{-d}\right)^{-d} \frac{1+\sigma_{1}}{\left(1-\sigma_{1}\right)^{2}} h_{k_{1}}^{1-\lambda^{2}-\delta_{1}} \\
& \leq\left(1-3^{-d}\right)^{-d} \frac{1+\sigma_{1}}{\left(1-\sigma_{1}\right)^{2}} 2 \theta_{1}^{-d} \operatorname{vol}(J)^{1-\lambda^{2}-2 \delta_{1}} h_{k_{1}}^{\delta_{1}} \\
& \leq \frac{1+\sigma_{1}}{\left(1-\sigma_{1}\right)^{2}} d^{-d / 2\left(1-\lambda^{2}-2 \delta_{1}\right)}|J|^{d-d \lambda^{2}-2 d \delta_{1}} .
\end{aligned}
$$

Possibility 1c. Finally, suppose that $\operatorname{vol}(J)<\left(\theta_{m} h_{k_{m}}^{1 / d}\right)^{d} / 2$. Recalling that for every $\ell=1, \ldots, M_{m}$, we have $\operatorname{vol}\left(J_{m, \ell}\right)=\left(\theta_{m} h_{k_{m}}^{1 / d}\right)^{d}$. Then, for any $m \geq 1$,

$$
\frac{M_{m}(J)}{M_{m}}=0 .
$$

In view of the results obtained for all possibilities of the Case 1, we conclude that for any $J \subseteq I^{d}$ verifying $I \subseteq J_{m-1, \ell_{0}}$, for $\ell_{0} \in\left\{1, \ldots, M_{m-1}\right\}$, we have the inequalities :

$$
\begin{aligned}
\frac{M_{1}(J)}{M_{1}} & \leq \frac{1+\sigma_{1}}{\left(1-\sigma_{1}\right)^{2}} d^{-d / 2\left(1-\lambda^{2}-2 \delta_{1}\right)}|J|^{d-d \lambda^{2}-2 d \delta_{1}}, & \\
\frac{M_{m}(J)}{M_{m}} & \leq \frac{1+\sigma_{m}}{\left(1-\sigma_{m}\right)^{2}} d^{-d / 2\left(1-\lambda^{2}-2 \delta_{m}\right)}|J|^{d-d \lambda^{2}-2 d \delta_{m}} & \text { for } m \geq 2 .
\end{aligned}
$$

Case 2. We now consider the case where $J$ is not necessarily a subset of $J_{m-1, \ell_{0}}$.

Possibility 2a. Assume that $\ell_{0} \in\left\{1, \ldots, M_{m-1}\right\}$ is the unique integer verifying $J \cap J_{m-1, \ell_{0}} \neq \varnothing$. Let $J^{\prime}:=$ $J \cap J_{m-1, \ell_{0}}$. It is easy to see that $M_{m}(J)=M_{m}\left(J^{\prime}\right)$. Moreover, $J^{\prime}$ verifies the assumptions of Case 1 . Therefore, 
we use (2.73) to obtain for any $m \geq 2$,

$$
\begin{aligned}
\frac{M_{m}(J)}{M_{m}} & \leq \frac{1+\sigma_{m}}{\left(1-\sigma_{m}\right)^{2}} d^{-d / 2\left(1-\lambda^{2}-2 \delta_{m}\right)}\left|J^{\prime}\right|^{d-d \lambda^{2}-2 d \delta_{m}} \\
& \leq \frac{1+\sigma_{m}}{\left(1-\sigma_{m}\right)^{2}} d^{-d / 2\left(1-\lambda^{2}-2 \delta_{m}\right)}|J|^{d-d \lambda^{2}-2 d \delta_{m}} .
\end{aligned}
$$

Possibility 2b. Assume that $J$ verifies $J \cap J_{m-1, \ell} \neq \varnothing$ for at least two different $\ell \in\left\{1, \ldots, M_{m-1}\right\}$. For any $m \geq 1$, let $H(m)$ denote the following property : $\forall T \subseteq I^{d}$,

$$
\frac{M_{m}(T)}{M_{m}} \leq \prod_{i=1}^{m}\left(\frac{1+\sigma_{i}}{1-\sigma_{i}}\right)^{2} d^{-\frac{d}{2}\left(1-\lambda^{2}-2 \Delta_{m}\right)}|T|^{d-d \lambda^{2}-2 d \Delta_{m}}, \text { where } \Delta_{m}:=\sum_{k=1}^{m} \delta_{k} .
$$

Remark 2.11. For $m=1$ and for all $T \subseteq I^{d},(2.72)$ implies (2.75). Moreover, for any $m \geq 1$, and $T$ verifying the assumptions of Case 1, (2.73) implies (2.75), and (2.75) is still true for any $m \geq 1$ verifying the assumptions of Case 2a.

Here and elsewhere, $\partial A$ denote the frontier of $A$, and $\stackrel{\circ}{\AA}$ the interior of $A$, for $A \subseteq \mathbb{R}^{k}, k \geq 1$.

Lemma 2.12. For $m \geq 2$, We assume that $H(m-1)$ holds and that $T \subseteq I^{d}$ is a hypercube such that

$$
\partial T \cap \bigcup_{\ell=1}^{M_{m-1}} \stackrel{\circ}{J}_{m-1, \ell}=\varnothing .
$$

Then, we have

$$
\frac{M_{m}(T)}{M_{m}} \leq \frac{1+(1 / 2) \sigma_{m}}{1+\sigma_{m}} \prod_{i=1}^{m}\left(\frac{1+\sigma_{i}}{1-\sigma_{i}}\right)^{2} d^{-\frac{d}{2}\left(1-\lambda^{2}-2 \Delta_{m-1}\right)}|T|^{d-d \lambda^{2}-2 d \Delta_{m-1}}
$$

Proof. By (2.76), it is easy to see that

$$
M_{m}(T)=\sum_{\substack{1 \leq \ell \leq M_{m-1} \\ J_{m}-1, \ell \subseteq T}} M_{m}\left(J_{m-1, \ell}\right) .
$$

Hence,

$$
\begin{aligned}
\frac{M_{m}(T)}{M_{m}} & =\sum_{\substack{1 \leq \ell \leq M_{m-1} \\
J_{m-1, \ell \subseteq T}}} \frac{M_{m}\left(J_{m-1, \ell}\right)}{M_{m}} \\
& \leq M_{m-1}(T) \times \max _{\substack{1 \leq \ell \leq M_{m-1} \\
J_{m-1, \ell \subseteq T}}}\left(\frac{M_{m}\left(J_{m-1, \ell}\right)}{M_{m}}\right) .
\end{aligned}
$$

Moreover, by (3ii), we know that $\operatorname{vol}\left(J_{m-1, \ell}\right)=L_{m-1}^{d} \geq h_{k_{m}}^{1-\lambda^{2}-\delta_{m}}$. Then, $J_{m-1, \ell}$ satisfies the assumptions of Case 1a. Therefore, we apply (2.67) with the formal replacement of $J$ by $J_{m-1, \ell}$ and $\operatorname{vol}(J)$ by $L_{m-1}$ and using 
(3iii),

$$
\begin{aligned}
\frac{M_{m}\left(J_{m-1, \ell}\right)}{M_{m}} & \leq \frac{1+\sigma_{m}}{\left(1-\sigma_{m}\right)^{2}} L_{m-1}^{d} \frac{1}{M_{m-1}\left(L_{m-1}^{*}\right)^{d}} \\
& \leq \frac{1+\sigma_{m}}{\left(1-\sigma_{m}\right)^{2}} \times\left(1+(1 / 2) \sigma_{m}\right) \times \frac{1}{M_{m-1}}
\end{aligned}
$$

Therefore, $H(m-1),(2.78)$, and (2.79) jointly imply that

$$
\begin{aligned}
\frac{M_{m}(T)}{M_{m}} & \leq \frac{M_{m-1}(T)}{M_{m-1}} \times\left(\frac{1+\sigma_{m}}{1-\sigma_{m}}\right)^{2} \times \frac{1+(1 / 2) \sigma_{m}}{1+\sigma_{m}} \\
& \leq \frac{1+(1 / 2) \sigma_{m}}{1+\sigma_{m}} \prod_{i=1}^{m}\left(\frac{1+\sigma_{i}}{1-\sigma_{i}}\right)^{2} d^{-\frac{d}{2}\left(1-\lambda^{2}-2 \Delta_{m-1}\right)}|T|^{d-d \lambda^{2}-2 d \Delta_{m-1}}
\end{aligned}
$$

This achieves the proof of (2.77).

Let $J^{\prime}$ denote the smallest hypercube which contains the sets $J_{m-1, \ell}$ verifying $J_{m-1, \ell} \cap J \neq \varnothing$, where $1 \leq \ell \leq M_{m-1}$, and $J^{\prime \prime}=J \cap J^{\prime}$. Then, we have

$$
M_{m}(J)=M_{m}\left(J^{\prime \prime}\right) \leq M_{m}\left(J^{\prime}\right)
$$

In the following, we use an inductive argument to prove (2.77) for $J$ verifying the assumptions of Case $2 \mathrm{~b}$. It is easy to see that $H(1)$ holds (see Rem. 2.11). Assume that $H(m-1)$ holds for any $m \geq 2$. We see that

$$
\partial J^{\prime} \cap \bigcup_{\ell=1}^{M_{m-1}} J_{m-1, \ell}=\varnothing .
$$

Apply Lemma 2.12 when $T=J^{\prime}$.

$$
\begin{aligned}
\frac{M_{m}\left(J^{\prime}\right)}{M_{m}} & \leq \frac{1+(1 / 2) \sigma_{m}}{1+\sigma_{m}} \prod_{i=1}^{m}\left(\frac{1+\sigma_{i}}{1-\sigma_{i}}\right)^{2} d^{-\frac{d}{2}\left(1-\lambda^{2}-2 \Delta_{m-1}\right)}\left|J^{\prime}\right|^{d-d \lambda^{2}-2 d \Delta_{m-1}} \\
& \leq \frac{1+(1 / 2) \sigma_{m}}{1+\sigma_{m}} \prod_{i=1}^{m}\left(\frac{1+\sigma_{i}}{1-\sigma_{i}}\right)^{2}\left(\operatorname{vol}(J) \frac{\operatorname{vol}\left(J^{\prime}\right)}{\operatorname{vol}(J)}\right)^{1-\lambda^{2}-2 \Delta_{m-1}} .
\end{aligned}
$$

But

$$
\operatorname{vol}\left(J^{\prime}\right) \leq\left[(\operatorname{vol}(J))^{1 / d}+2 L_{m-1}\right]^{d} \leq \sum_{k=0}^{d}\left(\begin{array}{l}
d \\
k
\end{array}\right) 2^{d-k} \operatorname{vol}(J)^{k / d} L_{m-1}^{d-k}
$$

Assume first that $\operatorname{vol}(J) \geq h_{k_{m-1}}$. (2.82) and (2.49) jointly imply that

$$
\begin{aligned}
\frac{\operatorname{vol}\left(J^{\prime}\right)}{\operatorname{vol}(J)} & \leq \sum_{k=0}^{d}\left(\begin{array}{l}
d \\
k
\end{array}\right) 2^{d-k} \frac{L_{m-1}^{d-k}}{\operatorname{vol}(J)^{(d-k) / d}} \\
& \leq \sum_{k=0}^{d}\left(\begin{array}{l}
d \\
k
\end{array}\right) 2^{d-k} \theta_{m-1}^{d-k} \leq\left(1+2 \theta_{m-1}\right)^{d}
\end{aligned}
$$


This last inequality, combined with (2.80), (2.81) and (2iii), imply that

$$
\frac{M_{m}(J)}{M_{m}} \leq \frac{M_{m}\left(J^{\prime}\right)}{M_{m}} \leq \prod_{i=1}^{m}\left(\frac{1+\sigma_{i}}{1-\sigma_{i}}\right)^{2} d^{-\frac{d}{2}\left(1-\lambda^{2}-2 \Delta_{m-1}\right)}|J|^{d-d \lambda^{2}-2 d \Delta_{m-1}} .
$$

Now assume that $\operatorname{vol}(J)<h_{k_{m-1}}$. Remembering that $\operatorname{vol}(J)>L_{m-1}^{d},(2.82)$ implies that

$$
\begin{aligned}
\frac{\operatorname{vol}\left(J^{\prime}\right)}{\operatorname{vol}(J)} & \leq \sum_{k=0}^{d}\left(\begin{array}{l}
d \\
k
\end{array}\right) 2^{d-k} \frac{L_{m-1}^{d-k}}{\operatorname{vol}(J)^{\frac{d-k}{d}}} \\
& \leq \sum_{k=0}^{d}\left(\begin{array}{l}
d \\
k
\end{array}\right) 2^{d-k} \leq 3^{d} .
\end{aligned}
$$

Combining (2.80), (2.81), (2.84), and (2iv), we get

$$
\frac{M_{m}(J)}{M_{m}} \leq \frac{M_{m}\left(J^{\prime}\right)}{M_{m}} \leq \prod_{i=1}^{m}\left(\frac{1+\sigma_{i}}{1-\sigma_{i}}\right)^{2} d^{-\frac{d}{2}\left(1-\lambda^{2}-2 \Delta_{m-1}\right)}|J|^{d-d \lambda^{2}-2 d \Delta_{m-1}} .
$$

Hence, (2.77) is verified for J verifying the assumptions of the Case $2 \mathrm{~b}$.

Case 3. We now consider the case where $J \cap J_{m-1, \ell}=\varnothing$ for any $\ell \in\left\{1, \ldots, M_{m-1}\right\}$. Observe that

$$
\frac{M_{m}(J)}{M_{m}}=0
$$

This last statement implies (2.77) for any $m \geq 1$.

SteP 3 : The Hausdorff dimension of $L_{\Lambda}$. Assertions (2.83) (2.85) and (2.86), combined with Remark 2.11 entail that $H(m)$ is true for any $m \geq 1$. By (1ii), we conclude that for any hypercube $J \subseteq I^{d}$, for any $m \geq 1$,

$$
M_{m}(J) \leq 2 d^{-d / 2\left(1-\lambda^{2}-2 \Delta_{m-1}\right)}|J|^{d\left(1-\lambda^{2}\right)-2 d \Delta_{m-1}} M_{m}
$$

Making use of Fact 2.2, with $A$ as previously described, $\Delta=1, d=2$ and $c=d\left(1-\lambda^{2}\right)-2 d \Delta_{m-1}$, we get $\operatorname{dim} A \geq d\left(1-\lambda^{2}\right)-2 d \Delta_{m-1}$. All that remains is to show that

$$
A=\bigcap_{m=1}^{\infty} E_{m} \subseteq L\left(\Theta_{\varphi}\right)
$$

Observe that $L_{m}=\theta_{m} h_{k_{m}}^{1 / d}<h_{k_{m}}^{1 / d}$. The fact that $k_{m}>k_{2}\left(\varepsilon_{m}, \sigma_{m}, \delta_{m}\right)>k_{0}\left(\varepsilon_{m}\right)$ implies by Lemma 2.7 that for any $m \geq 1, \mathbb{N}\left(L_{m}, W_{k_{m}, \varphi}\left(\varepsilon_{m}\right)\right) \subseteq U_{k_{m}, \varphi}\left(2 \varepsilon_{m}\right)$. But, (2.50), (2.28) and (2.29) allow us to say that for any $m \geq 1, E_{m} \subseteq \mathbb{N}\left(L_{m}, W_{k_{m}, \varphi}\left(\varepsilon_{m}\right)\right)$. This means that for any $m \geq 1$, for any $\mathbf{t} \in E_{m}$,

$$
\left\|\Theta_{h_{k_{m}}, \mathbf{t}}-\Theta_{\varphi}\right\|_{\mathcal{F}} \leq 2 \varepsilon_{m} .
$$

Since $A=\bigcap_{m=1}^{\infty} E_{m}$, this last inequality is verified for any $\mathbf{t} \in A$ and any $m \geq 1$. Finally, since $\varepsilon_{m} \rightarrow 0$ when $m \rightarrow \infty$, we obtain that $A \subseteq L\left(\Theta_{\varphi}\right)$. Hence, for all $\Lambda \in(0,1)$, we have $\operatorname{dim} L_{\Lambda} \geq d\left(1-\Lambda^{2}\right)$. This combined with (2.1) leads to (1.11). The proof of Theorem 1.1 is therefore achieved. 


\section{REFERENCES}

[1] M. Arcones, The large deviation principle of stochastic processes. II. Theory Probab. Appl. 48 (2003) 19-44.

[2] H. Chernoff, A measure of asymptotic efficiency for tests of a hypothesis based on the sum of observations. Ann. Math. Statistics 23 (1952) 493-507.

[3] P. Deheuvels and M.A. Lifshits, On the Hausdorff dimension of the set generated by exceptional oscillations of a Wiener process. Studia. Sci. Math. Hungar. 33 (1997) 75-110.

[4] P. Deheuvels and D.M. Mason, Random fractals generated by oscillations of processes with stationary and independent increments. Probability in Banach Spaces. 9 (1994) 73-90. (J. Hoffman-Jørgensen, J. Kuelbs and M.B. Marcus, eds.)

[5] P. Deheuvels and D.M. Mason, On the fractal nature of empirical increments. Ann. Probab. 23 (1995) 355-387.

[6] Z. Dindar, On the Hausdorff dimension of the set generated by exceptional oscillations of a two-parameter Wiener process. $J$. Multivariate Anal. 79 (2001) 52-70.

[7] K.J. Falconer, The Geometry of Fractal Sets. Cambridge University Press, Cambridge (1985).

[8] P. Lévy, Théorie de l'addition des variables aléatoires, Gauthier-Villars et Cie (1937)

[9] D.M. Mason, A uniform functional law of the logarithm for a local Gaussian process. Progress in Probability 55 (2003) 135-151.

[10] D.M. Mason, A uniform functional law of the logarithm for the local empirical process. Ann. Probab. 32 (2004) 1391-1418.

[11] S. Orey and S.J. Taylor, How often on a Brownian path does the law of the iterated logarithm fail? Proc. London Math. Soc. 28 (1974) 174-192.

[12] M. Schilder, Some asymptotic formulas for Wiener integrals. Trans. Amer. Math. Soc. 125 (1966) 63-85.

[13] M. Talagrand, Sharper bounds for gaussian and empirical processes. Ann. Probab. 22 (1994) $28-76$.

[14] A.W. van der Vaart and A.J. Wellner, Weak convergence and Empirical Processes. Springer, New-York (1996). 